\section{Harmonic ultrasonography for the detection of microlithiasis in the gallbladder}

\author{
Chul Soon Choi', You Jin Ku', Dae Young Yoon', Eun Joo Yun', Young Lan Seo', \\ Kyoung Ja Lim', Sora Baek', Sang Hoon Bae', Eun Sook Nam² \\ Departments of ${ }^{1}$ Radiology and ${ }^{2}$ Pathology, Kangdong Sacred Heart Hospital, Hallym \\ University College of Medicine, Seoul, Korea
}

Purpose: To validate the use of harmonic ultrasonography (US) in the detection of gallbladder microlithiasis.

Methods: From November 30, 2012, to January 18, 2014, fundamental US (FUS) and harmonic US with a high background noise (HUS-N) were performed for evaluation of gallbladder during the routine abdomen US. During the US, a dot-like stone (or stones) with Brownian motion was regarded as a positive finding of microlithiasis. Fifty-five patients with microlithiasis in the gallbladder detected on US were enrolled as the subjects of a retrospective review. With respect to the obtained images, two abdominal radiologists independently scored the conspicuity of gallbladder microlithiasis on FUS and HUS-N by using a 4-grade scale. The statistical analysis employed a kappa test and a Wilcoxon rank-sum test.

Results: For FUS, the conspicuity grades of gallbladder microlithiasis were G1 in 25 and 37, G2 in 21 and 9, G3 in 6 and 6, G4 in 3 and 3 patients, while HUS-N showed G1 in 0 and 0, G2 in 3 and 2, G3 in 12 and 15, and G4 in 40 and 38 patients, respectively, by each of the two radiologists. The kappa value was 0.633 for FUS between the two radiologists and 0.708 for HUS-N. HUS-N showed better conspicuity of gallbladder microlithiasis than FUS with significant P-values of less than 0.001 and 0.001 for the two radiologists, respectively.

Conclusion: Compared with FUS, HUS-N enables better detection of microlithiasis in the gallbladder.

Keywords: Gallbladder; Cholecystolithiasis; Ultrasonography

\section{Introduction}

Microlithiasis is defined as small stones (less than $3 \mathrm{~mm}$ ) in the gallbladder and is also referred to as sludge, biliary sand, biliary sediment, microcrystalline disease, pseudolithiasis, and reversible choledocholithiasis. They can be calcium crystals, cholesterol, or crystals of a mixed type [1-3]. Microlithiasis in the gallbladder is rarely detected on ultrasonography (US) or on other imaging modalities including multidetector computed tomography (CT) and magnetic resonance imaging (MRI) $[1,4]$. However, microlithiasis can cause nonspecific gallbladder and biliary pain $[5,6]$. Most

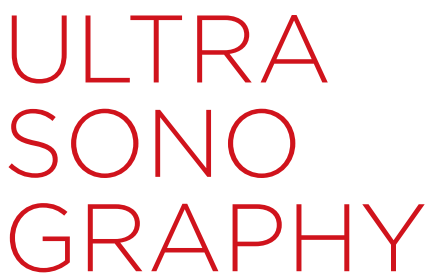

ORIGINAL ARTICLE

http://dx.doi.org/10.14366/usg.14024 pISSN: 2288-5919・elSSN: 2288-5943 Ultrasonography 2014;33:275-282

Received: May 23, 2014

Revised: June 23, 2014

Accepted: June 28, 2014

Correspondence to:

Chul Soon Choi, MD, PhD, Department of Radiology, Kangdong Sacred Heart Hospital, Hallym University College of Medicine, 150 Seongan-ro, Gangdong gu, Seoul 134-701, Korea

Tel. $+82-2-2224-2656$

Fax. +82-2-488-7370

E-mail: chulsoon56@gmail.com

This is an Open Access article distributed under the terms of the Creative Commons Attribution NonCommercial License (http://creativecommons.org/ licenses/by-nc/3.0/) which permits unrestricted noncommercial use, distribution, and reproduction in any medium, provided the original work is properly cited.

Copyright @ 2014 Korean Society of Ultrasound in Medicine (KSUM)

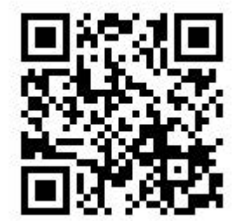

How to cite this article:

Choi CS, Ku YJ, Yoon DY, Yun EJ, Seo YL, Lim $\mathrm{KJ}$, et al. Harmonic ultrasonography for the detection of microlithiasis in the gallbladder. Ultrasonography. 2014 0ct;33(4):275-282. 
importantly, microlithiasis can cause acute idiopathic pancreatitis [5]. To date, these types of microlithiasis have not been described on radiological imaging in the global medical literature. Only visible microlithiasis has been described using endoscopic US that depicts the microliths through the duodenal wall $[1,7]$.

In contrast to fundamental US (FUS), the waves of harmonic US have a nonlinear double frequency and half wavelength although the pulse length of a harmonic US wave is the same as that of an FUS wave. The short wavelength might be one of the factors enabling the detection of smaller targets in the gallbladder. A major use of harmonic US previously described is better detection of microbubble contrast agents and detection of fluid in the lumens or cysts. It yields fewer artifacts, particularly side-lobe artifacts, and has an increased signal-to-noise ratio; further, it has an increased lateral resolution in the case of a narrow bandwidth and a better spatial resolution in the case of a narrow wavelength; therefore, in the abdomen and the pelvis, a better contrast can be achieved $[8,9]$.

We hypothesized that harmonic US would be better than FUS for the detection of microlithiasis (less than $3 \mathrm{~mm}$ ) in the gallbladder. In this study, we compared the use of the techniques of FUS and harmonic US with a high background noise (HUS-N) in the detection of gallbladder microlithiasis. To optimize the visualization of small echogenic materials, a high background noise was made by increasing the overall gain control to obtain the higher intensities of echoes from the gallbladder during the harmonic US. The purpose of this study was to validate the harmonic US in the detection of gallbladder microlithiasis.

\section{Materials and Methods}

This retrospective study was approved by the Ethics Review Board of our hospital; the Board waived the requirement of informed consent from the patients because it was not an invasive study and the US examinations were performed as routine studies of the abdomen.

The study period was from November 30, 2012, to January 19, 2014. During this period, one abdominal radiologist who had 29 years of experience in abdominal US performed all of the US examinations of the abdomens of patients who were referred to him (the total number of examinations of abdominal US performed by this radiologist during the study period was 1,914 ). The US equipment was an iU 22 (Philips Medical System, Bothell, WA, USA) with a 2- to 5-MHz convex array transducer for abdominal imaging and the manufacturer's preloaded software XRES and sonoCT. The other equipment consisted of an HDI 5000 (Advanced Technology Laboratory, Bothell) with a 2- to $5-\mathrm{MHz}$ abdominal sector transducer with sonoCT turned on and a LOGIQ9 (General Electric, Milwaukee, WI, USA) with a 1- to 6-MHz abdominal sector transducer. The initial routine abdominal FUS, including the liver, the pancreas, the spleen, both kidneys, and the bowels, was performed in every patient. Finally, the radiologist observed the gallbladder to search for microlithiasis and then performed HUS-N irrespective of whether microlithiasis was present or not. High background noise was used by increasing the overall two-dimensional gain until the background echoes were sufficiently high to view the definition of the abdominal organs with minimal difficulty. Then, the background noise was gradually decreased until the microliths were clearly or vaguely visible. If microliths-a dot or dots-were noted, we observed them until their Brownian motion was apparent and recorded this as a positive finding [7]. However, with LOGIC 9, we initially performed default harmonic imaging for the abdomen, and particularly, for the gallbladder, we increased the overall gain until the microliths were observed. Further, irrespective of the presence of microliths, we switched equipment to fundamental imaging for the visibility of microliths.

The exclusion criteria for our data were combined large (larger than $5 \mathrm{~mm}$ ) gallstones [10], gallbladder sludge observed on fundamental imaging (which was outside of our study's scope even if it was made of crystals, and was easily and frequently observed), and acute cholecystitis (Fig. 1). The US criteria for acute cholecystitis were Murphy's sign or moderate-to-severe right upper quadrant (gallbladder area) pain and thickening of the gallbladder wall by more than $3 \mathrm{~mm}$ [11]. However, we did not count the number of these patients because they were not the focus of this study. During the period, we found 55 cases of microlithiasis without large stones, gallbladder sludge, or any evidence of acute cholecystitis on the US examinations. The male-to-female ratio was 32:23 with an average age of $53.3 \pm 12.9$ years. The complaints of the patients were mainly nonspecific (40 cases) - there was only one case of acute idiopathic

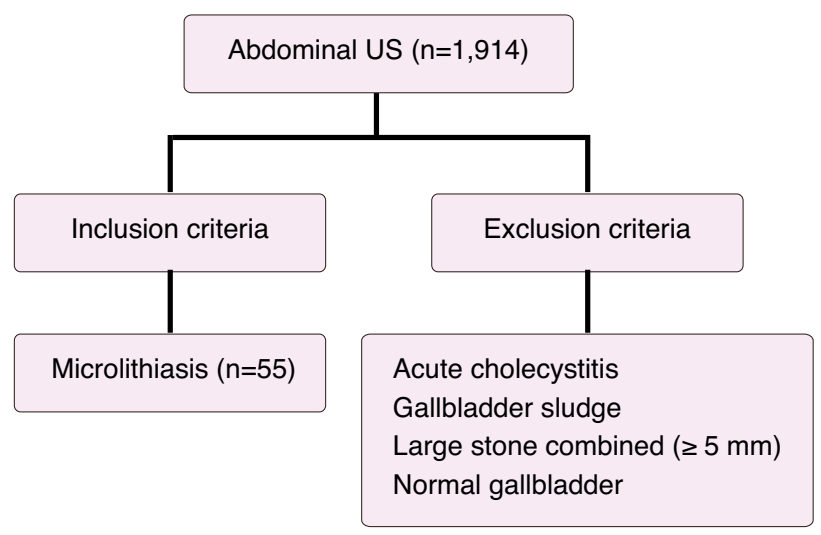

Fig. 1. Flowchart of the inclusion and exclusion criteria of our data sampling. US, ultrasonography. 
pancreatitis, and 14 cases showed gallbladder-related symptoms (Table 1). Eight patients who visited our hospital for a health checkup showed microlithiasis without any of its symptoms or signs (Table 1). Another 32 patients had specific problems not related to the gallbladder or the biliary trees.

After copying the microlithiasis images in the custom folder of the radiologist, two radiologists who had 29 and 19 years of experience, respectively, in abdominal radiology independently interpreted the images by using a picture archive communication system (Infinit, Seoul, Korea). There were two sessions. One consisted of the FUS images 4 weeks after the images were obtained. The sequence of interpretation was not the order in which they were obtained but was decided randomly by using a random number table. Four weeks after the FUS image interpretation, a second session for the interpretation of the HUS-N images was held independently by the two radiologists by using a random order of the images based on a random number table as in the session for the FUS image interpretation. All of the images were interpreted subjectively with the use of a 4-grade scale as follows: grade 1, no visible microlith; grade 2, vaguely visible-not definitely present; grade 3 , not clearly visible but present; and grade 4, clearly visible microliths.

After grading the conspicuity of the microliths on both sets of images (FUS and HUS-N), the kappa values were calculated for the grades assigned by the two radiologists, by using the kappa test calculator found at the website (http://vassarstats.net/kappa.html). We considered a value greater than 0.81 to represent an almost perfect agreement and the values of $0.61-0.81$ and $0.41-0.60$ to represent a substantial or moderate agreement. Values of less than
0.40 represented a fair agreement [12]. The statistical analysis was performed using a Wilcoxon rank-sum test (IBM SPSS ver. 19.0, IBM Co., Armonk, NY, USA) for each radiologist's interpretation. A $\mathrm{P}$-value of less than 0.05 was considered statistically significant.

Table 1. Symptoms of the patients with microcrystals resulting in referral for ultrasonography

\begin{tabular}{lc}
\hline \multicolumn{1}{c}{ Gallbladder-related symptoms } & No. of patients $(\mathrm{n}=55)$ \\
\hline RUQ pain (cholecystectomy) & $14(6)$ \\
Gallbladder-unrelated symptoms or causes & 1 \\
Acute idiopathic pancreatitis & 8 \\
Health check-up & 8 \\
LFT elevation & 2 \\
Diarrhea & 1 \\
Epigastric pain & 2 \\
Dyspepsia & 3 \\
Renal disease suspected & 3 \\
Fever & 3 \\
Known gallbladder stone follow-up & 4 \\
HBV carrier follow-up & 2 \\
HCV carrier follow-up & 1 \\
Alcoholics & 2 \\
Abdominal pain (lower abdomen, RLQ) & 1 \\
Known liver abscess follow-up &
\end{tabular}

RUQ, right upper quadrant; LFT, liver function test; HBV, hepatitis B virus; HCV, hepatitis C virus; RLQ, right lower quadrant.

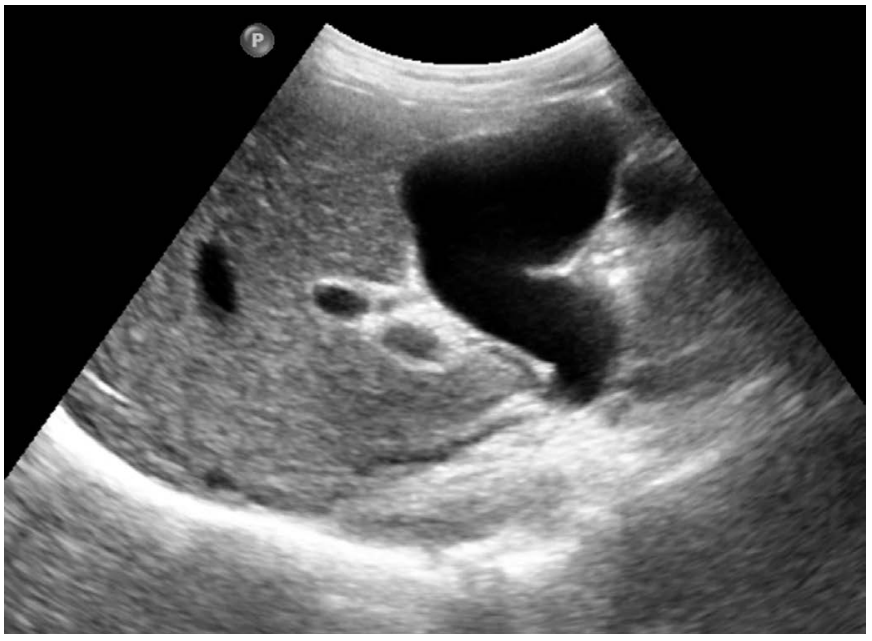

A

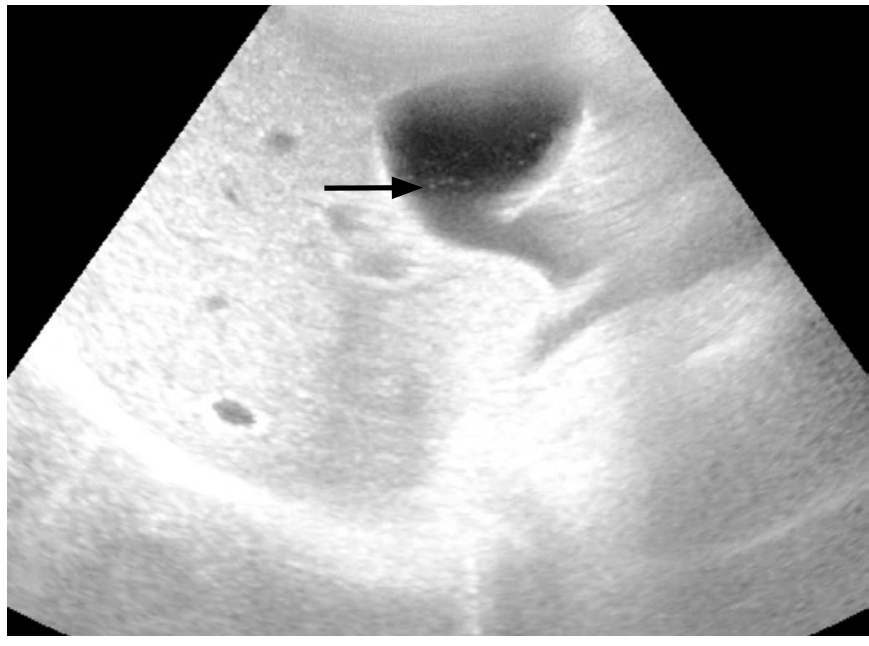

Fig. 2. A 58-year-old man with diarrhea.

A. Fundamental ultrasonography shows no demonstrable stones or microlithiasis in the gallbladder lumen. B. Harmonic ultrasonography with a high background noise clearly shows tiny echogenic materials suggesting microliths (arrow) in the gallbladder lumen. 


\section{Results}

The conspicuity grades of the gallbladder microlithiasis are summarized in Table 2, and the concordances of interpretation between the two radiologists were substantial on FUS (the unweighted kappa value was 0.651 with a standard error [SE] of 0.085 ) and on HUS-N, the unweighted kappa value was 0.708 with an SE of 0.103. HUS-N showed more conspicuous microliths than FUS (Figs. 2, 3). (The Wilcoxon rank-sum test revealed $P<0.001$ for both radiologists with Z-values of 6.09 and 6.05 , respectively.) A case with a history of five episodes of acute idiopathic pancreatitis showed microlithiasis on the last examination on HUS-N, while there were no such abnormal echogenic spots on the FUS (Fig. 4).

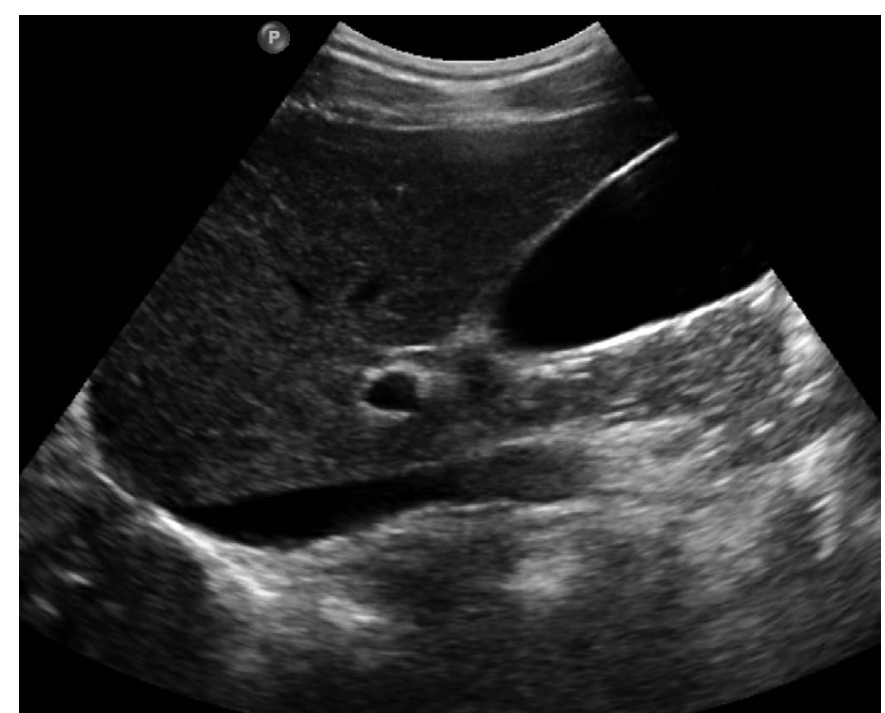

A

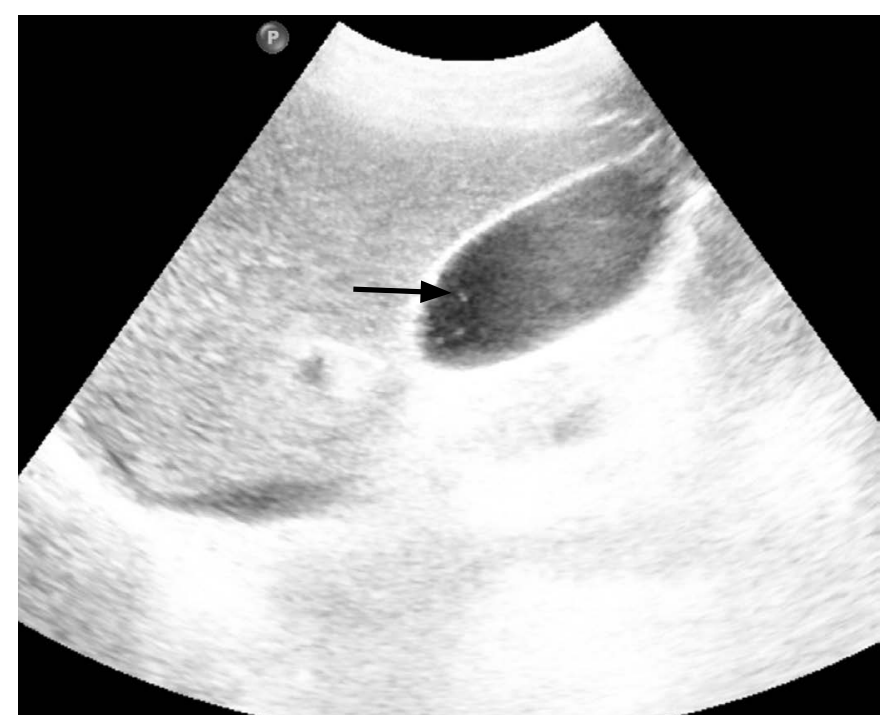

Table 2. Conspicuity of two different ultrasonography techniques in the assessment of microcrystals in the gallbladder

\begin{tabular}{cccccc}
\hline \multirow{2}{*}{ Grade } & \multicolumn{2}{c}{ FUS $(\mathrm{n}=55)$} & & \multicolumn{2}{c}{ HUS-N } \\
\cline { 2 - 3 } \cline { 5 - 6 } & Reader 1 & Reader 2 & & Reader 1 & Reader 2 \\
\hline 1 & 25 & 37 & & 0 & 0 \\
2 & 21 & 9 & 3 & 2 \\
3 & 6 & 6 & & 12 & 15 \\
4 & 3 & 3 & & 40 & 38 \\
\hline
\end{tabular}

Kappa value with linear weighting: FUS, 0.633 with $95 \%$ confidence interval from 0.478 to 0.789 ; HUS-N, 0.708 with $95 \%$ confidence interval from 0.566 to 0.925 .

FUS, fundamental ultrasonography; HUS-N, harmonic ultrasonography with a high background noise.

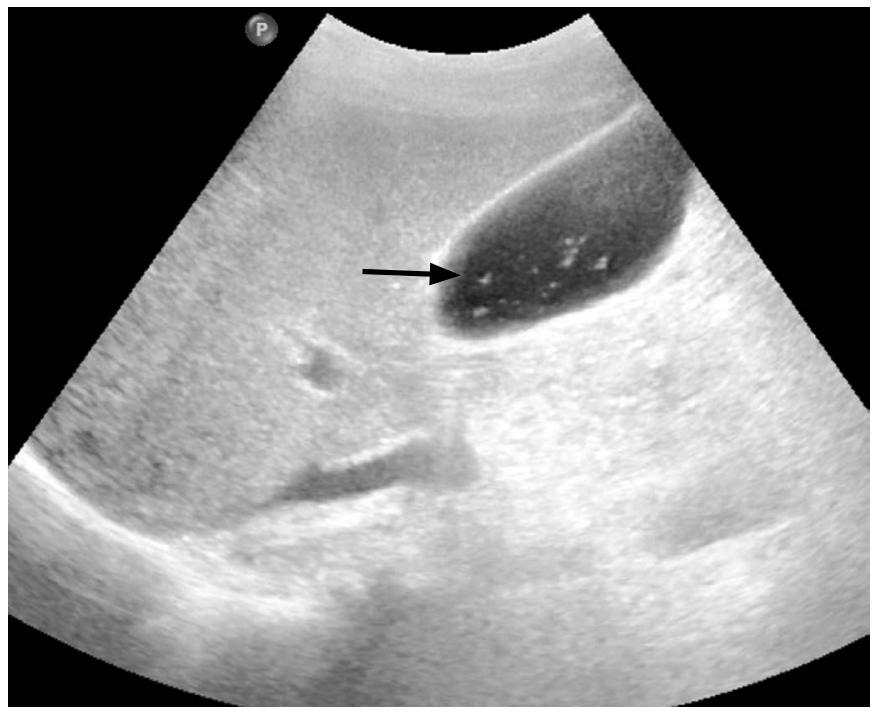

B

Fig. 3. A 59-year-old man with right upper abdominal pain. A. There are no stones in the lumen and no microliths on fundamental ultrasonography. B. Harmonic ultrasonography with a high background noise clearly demonstrates microliths (arrow) in the lumen with grade 4 conspicuity. C. Fundamental images with a high background noise shows grade 3 conspicuity of microliths (arrow). 
Among the subjected patients, six patients developed aggravation of the right upper abdominal pain and tenderness later, and cholecystectomies were performed revealing 3 cases of chronic cholecystitis, while the other 3 cases were of acute cholecystitis. However, of these 6 cases, 4 cases showed no stone in the gallbladder lumen after cholecystectomy. All of these cases showed grade 4 conspicuity of gallbladder microlithiasis on the HUS-N.

\section{Discussion}

Microlithiasis consisting of cholesterol monohydrate or calcium bilirubinate or mixed crystals has not been well known to radiologists due to poor visualization on the transabdominal US, CT, and MRI $[1,4]$. In the Meltzer-Lyons test, which is the most sensitive method for confirming microlithiasis, gallbladder contraction was induced by the injection of cholecystokinin, and duodenal fluid was

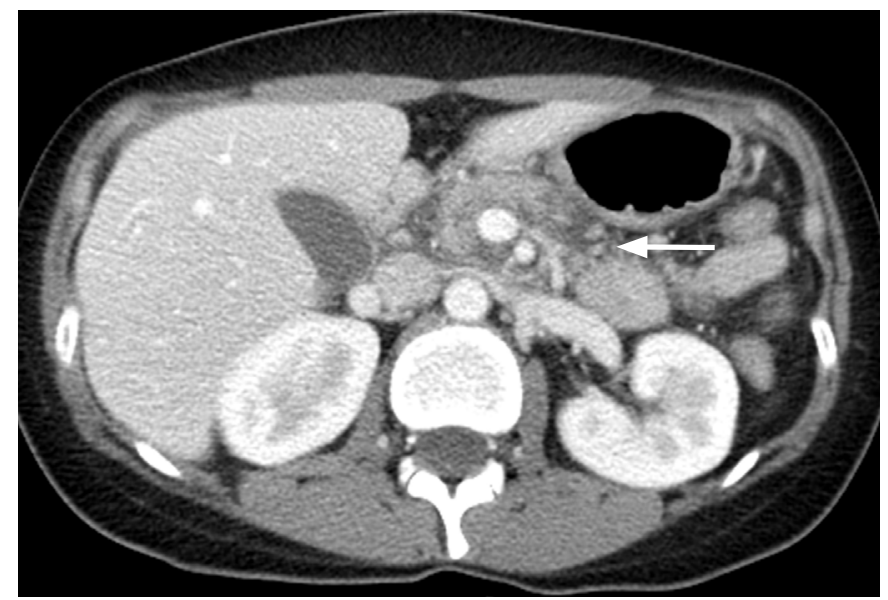

A

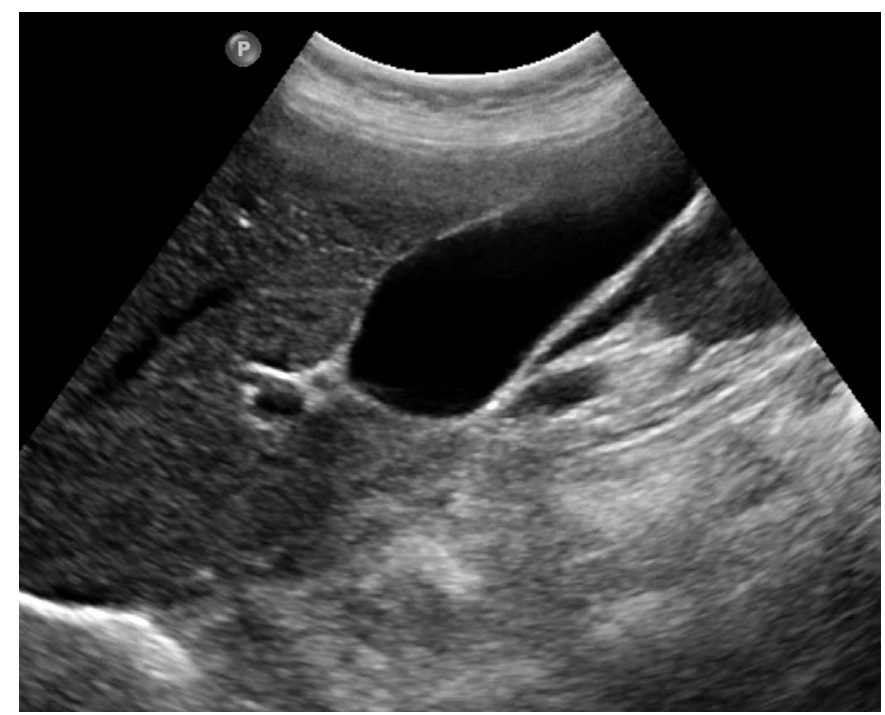

B

e-ultrasonography.org

Ultrasonography 33(4), October 2014 collected by endoscopy or cannulation through the ampulla of Vater. The fluid was centrifuged at $3,000 \mathrm{~g}$, and the precipitates in the test tube base were examined with polarized microscopy to determine whether there were crystals present or not [13-15]. Sometimes, without inducing gallbladder contraction with cholecystokinin, duodenal fluid collection could be performed with endoscopy or cannulation of the bile duct, and the fluid was then analyzed in the same way $[16,17]$. The only radiological method for visualizing microlithiasis is endoscopic US, which shows the microliths through the duodenal wall $[1,7]$. As far as we know, there have been no reports on the imaging of microlithiasis with transabdominal US, because the usual fundamental imaging during routine abdomen US cannot visualize microlithiasis well in the gallbladder.

Microlithiasis is important in medicine because microliths induce biliary pain and discomfort without definite gallbladder stones [5]. In two limited studies, one-third of acalculous gallbladder pain

Fig. 4. A 31-year-old woman having 5 episodes of acute recurrent idiopathic pancreatitis.

A. Axial plane of contrast-enhanced abdomen computed tomography shows the swelling of pancreas and peripancreatic fluid (arrow). B. Fundamental ultrasonography shows no stones in the gallbladder lumen. C. Harmonic ultrasonography with a high background noise clearly shows the microliths (arrow) in the gallbladder lumen. Cholecystectomy was recommended, but the patient refused.

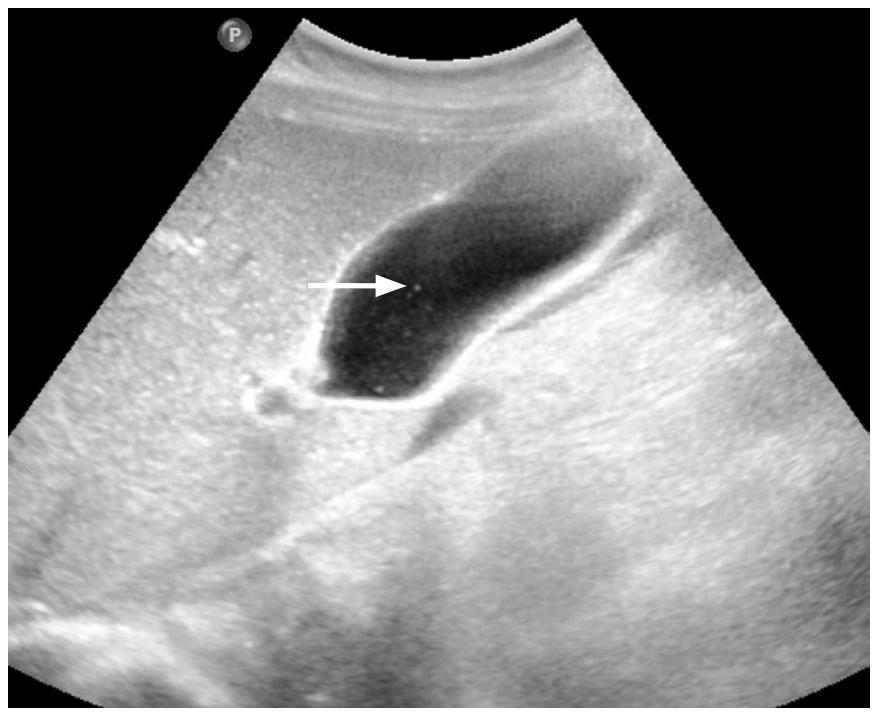

C 
patients showed microlithiasis in the bile juice obtained with the endoscopic duodenal fluid after a cholecystokinin injection, and nearly all the gallbladder specimens revealed chronic inflammation and gallbladder fluid-contained microlithiasis on a microscopic bile examination after cholecystectomy $[18,19]$. In our study, 14 patients who had gallbladder symptoms exhibited grade 4 microlithiasis on HUS-N, including the six patients who were confirmed to have chronic or acute cholecystitis after cholecystectomy in spite of the negative preoperative US feature for cholecystitis. Gallbladder microlithiasis can also cause acute idiopathic pancreatitis by passing through the cystic duct inducing a transient obstruction of the pancreatic duct $[6,20]$. A substantial portion $(13 \%-22 \%)$ of acute idiopathic pancreatitis can be explained by microlithiasis $[1,5,13]$.

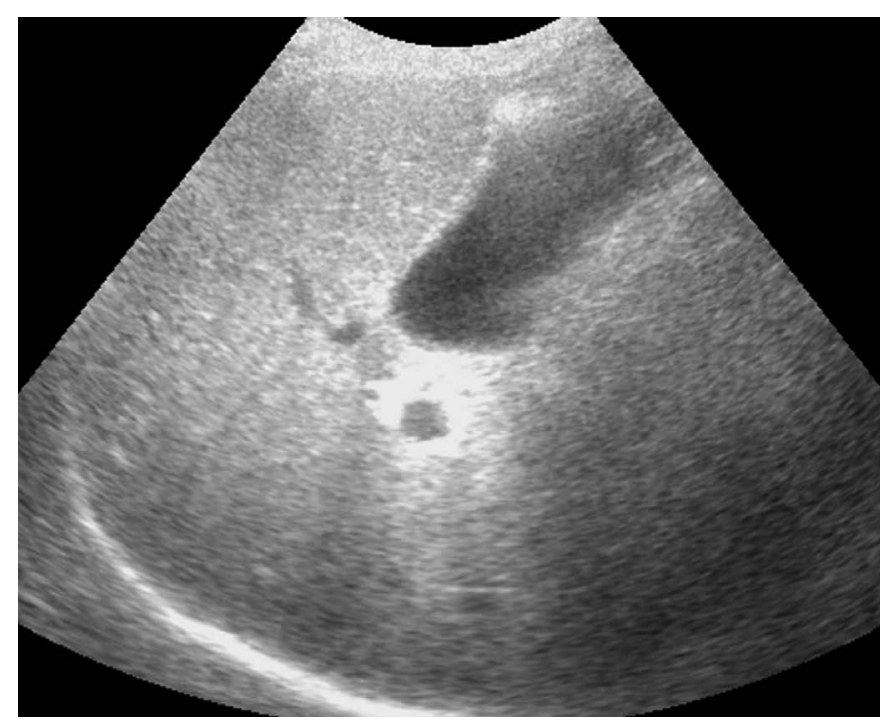

A

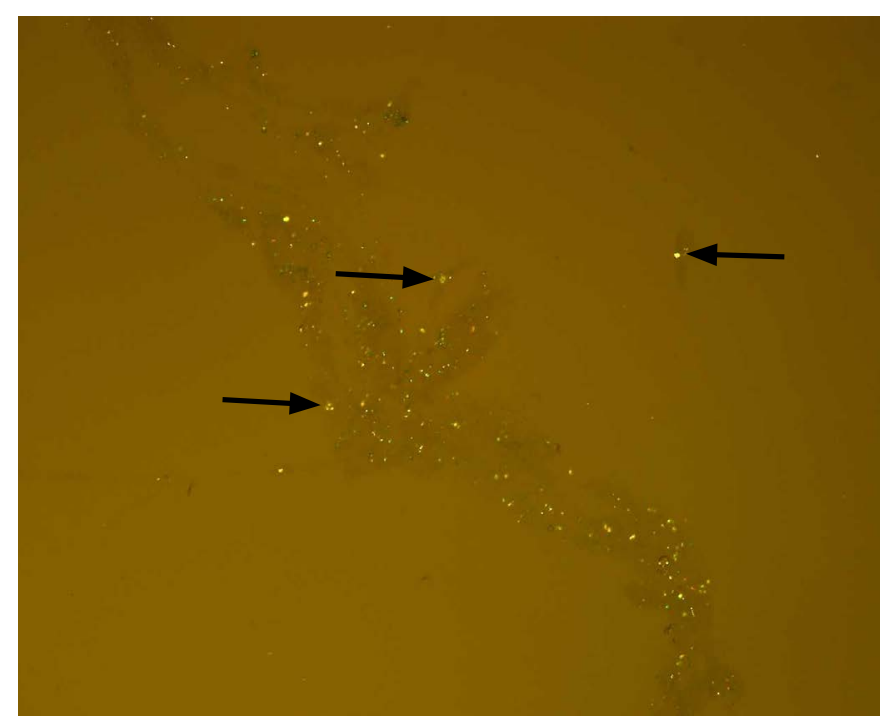

During the image-obtaining period, we performed US for acute idiopathic pancreatitis in 11 patients who were included in the initial 1,914 patients. Among them, we found two patients with gallbladder sludge and one microlithiasis patient.

The second harmonic US has a nonlinear double-wave frequency with the same pulse length as compared to FUS. It yields fewer artifacts, particularly side-lobe artifacts, and has an increased signalto-noise ratio, and this has an increased lateral resolution due to a narrow bandwidth and a better spatial resolution due to a narrow wavelength; therefore, in the abdomen and the pelvis, a better contrast can be achieved $[8,9]$. The second harmonic waves occur while passing through the medium after an incident wave of high intensity is sent through the tissue medium from the transducer.

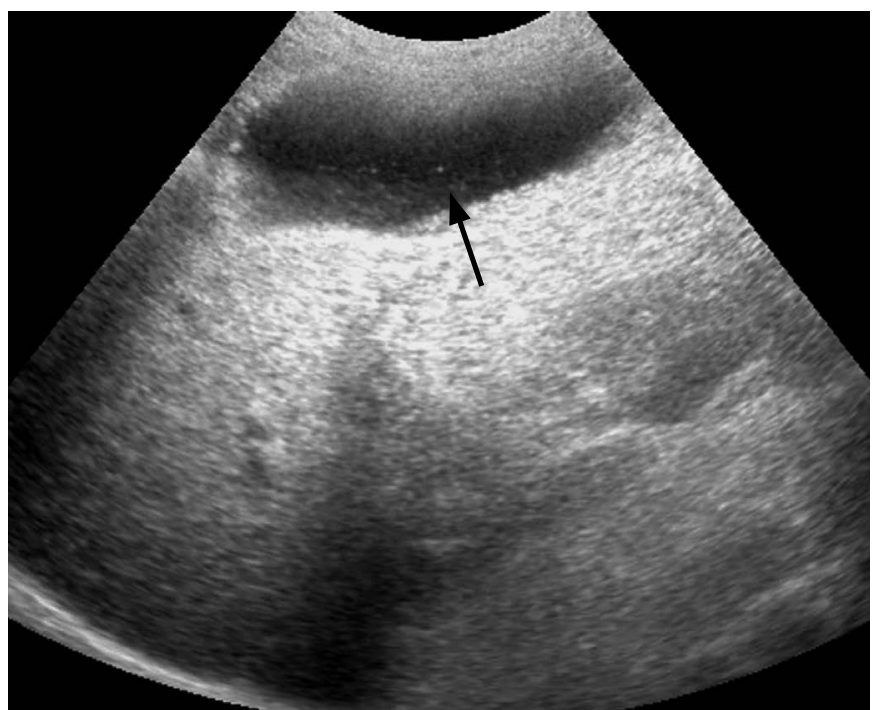

B

Fig. 5. A 76-year-old woman with acute cholecystitis.

A. Fundamental ultrasonography shows no stones in the gallbladder lumen. B. Harmonic ultrasonography with a high background noise shows microlithiasis (arrow) in the gallbladder lumen. C. Gallbladder fluid was percutaneously aspirated and centrifuged. The precipitates show clear microcrystals with some colloid components (arrows) on polarized microscopy $(\times 200)$. 
Due to the short wavelength, harmonic waves provide a better spatial resolution, and the lateral resolution is also better due to the narrow bandwidth of these waves as compared to the fundamental waves $[8,9]$. HUS-N's superiority in detecting microlithiasis might mostly depend on the better spatial and lateral resolutions observed in this study. Another explanation is the superficial location of the gallbladder. Harmonic waves have the disadvantage of attenuating more rapidly in the medium than fundamental waves. The gallbladder is usually located in a superficial area, and the intensity of the harmonic waves could be preserved well during the transmission with an improved contrast resolution and a high signalto-noise ratio. The gallbladder is the storage organ for bile fluid. Microliths float in the bile fluid in the gallbladder lumen. The better contrast resolution and higher signal-to-noise ratio in harmonic images could make such imaging better for depicting microliths $[8,9]$. By increasing the background noise, the increased intensity of the echoes from the medium could degrade the overall image quality of the bile juice-containing gallbladder. However, the degradation of fluid images is less than that of the solid portion of the microliths; therefore, it might produce a better visualization of microlithiasis. However, these explanations are speculative on the basis of the literature, and all possible explanations should be tested.

There are several limitations of our study. First of all, there was no proof that microliths on US were true ones on microscopy, and this limitation resulted in patient selection bias. The patients of gallbladder microlithiasis were not sampled among patients in order to have true positive microlithiasis in the gallbladder; instead, among the 1,914 patients who were examined using abdominal US, we sampled the patients to show the findings suggestive of microlithiasis on US. It seemed to be unethical to aspirate the gallbladder content for the patients with no relevant symptoms or signs. After the completion of this study, we met one patient with acute cholecystitis showing microlithiasis on HUS-N but not on FUS. In this patient, we performed percutaneous transhepatic gallbladder drainage, and microcrystals were found in the centrifuged gallbladder fluid on the polarized microscopy (Fig. 5). We thought that this finding could be applied to the other cases enrolled in this study. A major portion of microlithiasis in our data was less than 1 $\mathrm{mm}$ as in the case shown in Fig. 5. The crystals in the precipitates were smaller than the microliths seen on the US in this case (Fig. $5 B, C)$. These might have been the particles of the crushed microlith after centrifugation. We did not know whether there were false negative cases. The first radiologist who graded the images was the radiologist who collected the microlithiasis images, and there was a potential bias during the image interpretation. To minimize the interpretation bias, interpretation sessions were separated one and two months after the imaging. To verify the results of our study, a cohort study targeting the gallbladder patients planning the cholecystectomy or percutaneous gallbladder drainage could be helpful. For a fairer comparison, FUS with high background noise could be used in a comparative study with HUS-N. From a practical perspective, however, high background noise is not adequate for the usual US examination of the abdomen because it degrades the overall image quality and is even poorer than HUS-N in the detection of microlithiasis as in the case shown in Fig. 3.

In conclusion, despite several biases in the study design, the HUS-N capability in various current US machines allowed a better visualization of microlithiasis in the gallbladder than FUS. Depending on the results of this study, harmonic imaging during the gallbladder US could be useful in the diagnosis of microlithiasis, which cannot be detected on conventional abdomen US.

ORCID: Chul Soon Choi: http://orcid.org/0000-0002-9548-6693; You Jin Ku: http:// orcid.org/0000-0002-6727-0289; Dae Young Yoon: http://orcid.org/0000-00031522-0236; Eun Joo Yun: http://orcid.org/0000-0002-7346-8187; Young Lan Seo: http://orcid.org/0000-0002-9599-6423; Kyoung Ja Lim: http://orcid.org/0000-00033161-2811; Sora Baek: http://orcid.org/0000-0001-6536-2385; Sang Hoon Bae: http://orcid.org/0000-0002-0697-9868; Eun Sook Nam: http://orcid.org/0000-00032246-6269

\section{Conflict of Interest}

No potential conflict of interest relevant to this article was reported.

\section{Acknowledgments}

This study was supported in part by the Research Fund of the Korean Society of Ultrasound in Medicine.

\section{References}

1. Levy MJ. The hunt for microlithiasis in idiopathic acute recurrent pancreatitis: should we abandon the search or intensify our efforts? Gastrointest Endosc 2002;55:286-293.

2. Houssin D, Castaing D, Lemoine J, Bismuth $H$. Microlithiasis of the gallbladder. Surg Gynecol Obstet 1983;157:20-24.

3. Abeysuriya V, Deen KI, Navarathne NM. Biliary microlithiasis, sludge, crystals, microcrystallization, and usefulness of assessment of nucleation time. Hepatobiliary Pancreat Dis Int 2010;9:248-253.

4. Rebner M, Ruggieri PM, Gross BH, Glazer GM. CT evaluation of intracholecystic bile. AJR Am J Roentgenol 1985;145:237-240.

5. Saraswat VA, Sharma BC, Agarwal DK, Kumar R, Negi TS, Tandon RK. Biliary microlithiasis in patients with idiopathic acute pancreatitis and unexplained biliary pain: response to therapy. J Gastroenterol Hepatol 2004;19:1206-1211.

6. Moskovitz M, Min TC, Gavaler JS. The microscopic examination of bile in patients with biliary pain and negative imaging tests. Am J Gastroenterol 1986;81:329-333. 
7. Dahan P, Andant C, Levy P, Amouyal P, Amouyal G, Dumont M, et al. Prospective evaluation of endoscopic ultrasonography and microscopic examination of duodenal bile in the diagnosis of cholecystolithiasis in 45 patients with normal conventional ultrasonography. Gut 1996;38:277-281.

8. Oktar SO, Yucel C, Ozdemir H, Uluturk A, Isik S. Comparison of conventional sonography, real-time compound sonography, tissue harmonic sonography, and tissue harmonic compound sonography of abdominal and pelvic lesions. AJR Am J Roentgenol 2003;181:1341-1347.

9. Shapiro RS, Wagreich J, Parsons RB, Stancato-Pasik A, Yeh HC, Lao R. Tissue harmonic imaging sonography: evaluation of image quality compared with conventional sonography. AJR Am J Roentgenol 1998;171:1203-1206.

10. Diehl AK, Holleman DR Jr, Chapman JB, Schwesinger WH, Kurtin WE. Gallstone size and risk of pancreatitis. Arch Intern Med 1997;157:1674-1678.

11. Cooperberg PL, Gibney RG. Imaging of the gallbladder, 1987. Radiology 1987;163:605-613.

12. Lee ES, Kim SH, Lee JY, Kim SJ, Kim MA, Lee JM, et al. Radiologist performance in differentiating polypoid early from advanced gastric cancer using specific CT criteria: emphasis on dimpling sign. AJR Am J Roentgenol 2009;193:1546-1555.

13. Ros E, Navarro S, Bru C, Garcia-Puges A, Valderrama R. Occult microlithiasis in 'idiopathic' acute pancreatitis: prevention of relapses by cholecystectomy or ursodeoxycholic acid therapy. Gastroenterology 1991;101:1701-1709.

14. Lee SP, Nicholls JF, Park HZ. Biliary sludge as a cause of acute pancreatitis. N Engl J Med 1992;326:589-593.

15. Neoptolemos JP, Davidson BR, Winder AF, Vallance D. Role of duodenal bile crystal analysis in the investigation of 'idiopathic' pancreatitis. Br I Surg 1988;75:450-453.

16. Kaw M, Brodmerkel GJ Jr. ERCP, biliary crystal analysis, and sphincter of Oddi manometry in idiopathic recurrent pancreatitis. Gastrointest Endosc 2002;55:157-162.

17. Buscail L, Escourrou J, Delvaux M, Guimbaud R, Nicolet T, Frexinos $J$, et al. Microscopic examination of bile directly collected during endoscopic cannulation of the papilla: utility in patients with suspected microlithiasis. Dig Dis Sci 1992;37:116-120.

18. Susann PW, Sheppard F, Baloga AJ. Detection of occult gallbladder disease by duodenal drainage collected endoscopically: a clinical and pathologic correlation. Am Surg 1985;51:162-165.

19. Porterfield G, Cheung LY, Berenson M. Detection of occult gallbladder disease by duodenal drainage. Am J Surg 1977;134: 702-704.

20. Chebli JM, Duarte Gaburri P, Meirelles de Souza AF, de Castro Ferreira LE, Andrade Chebli L, Ferrari AP Jr, et al. "Idiopathic" acute pancreatitis due to biliary sludge: prevention of relapses by endoscopic biliary sphincterotomy in high-risk patients. Am J Gastroenterol 2000;95:3008-3009. 